\title{
Morphology of Novel Chitosan Oligosaccharides Mod- ified Cross-linked Polyvinyl Alcohol Complex Foam (COS/cPVACF) Dressings with Fully Open-cell and Open-channel Microstructures via Active Molecules Cleaning Process for Wound Managements
}

\author{
Xiongxin Lei ${ }^{1,2}$, Guifeng Zhang ${ }^{1,2}$, Meng-Jen Yang ${ }^{1}$, Yuguang $\mathrm{Du}^{2}$ and Ching-Cheng Huang ${ }^{* 1,3}$ \\ ${ }^{1}$ PARSD Biomedical Material Research Center (PBMRC), Jiangsu \\ ${ }^{2}$ Institute of process engineering, Chinese Academy of Sciences, Beijing \\ ${ }^{3}$ Department of Biomedical Engineering, Taiwan \\ *Corresponding author: Ching-Cheng Huang, PARSD Biomedical Material Research Center (PBMRC), Jiangsu; Department of \\ Biomedical Engineering, Ming Chuan University, Taoyuan, Taiwan
}

\section{ARTICLE INFO}

Received: 蔧 April 16, 2019

Published: April 24, 2019

Citation: Xiongxin L, Guifeng Z, Meng-Jen Y, Yuguang D, Ching-Cheng Huang. Morphology of Novel Chitosan Oligosaccharides Modified Crosslinked Polyvinyl Alcohol Complex Foam (COS/cPVACF) Dressings with Fully Open-cell and Open-channel Microstructures via Active Molecules Cleaning Process for Wound Managements. Biomed J Sci \& Tech Res 17(3)-2019. BJSTR. MS.ID.003001.

\begin{abstract}
High permeability of protecting dressings such as cross-linked polyvinyl alcohol (cPVA) foam or polyurethane (PU) foam/film or non-woven dressings could be employed for the clinic application of wound management. However, polyurethane foam or nonwoven dressings showed poor cell or tissue anti-adhesion property. The cross-linked polyvinyl alcohol foam dressings could be used for wound management because of good cell or tissue anti-adhesion property. Furthermore, the permeable chitosan oligosaccharide modified cross-linked polyvinyl alcohol complex foam (COS/cPVACF) dressings could be designed and prepared for the clinic applications. Fully open-cell and open-channel Microstructures could be designed and formed within the new chitosan oligosaccharides modified cross-linked polyvinyl alcohol complex foam (COS/cPVACF) dressings. Morphology of the resulting chitosan oligosaccharides modified cross-linked polyvinyl alcohol complex foam (COS/CPVACF) dressings with fully open-cell and openchannel microstructures could be observed.
\end{abstract}

Keywords: Chitosan Oligosaccharide; Medical Device; Permeability; Dressings

Abbreviations: PU: polyurethane; cPVA: cross-Linked Polyvinyl Alcohol; cPVACF: crossLinked Polyvinyl Alcohol Complex Foam; COS: Chitosan Oligosaccharide; FTIR: Fourier Transform Infrared Spectroscopy; SEM: Scanning Electron Microscopy

\section{Introduction}

The design of medical devices could be applied and developed for new treatment proce-dures instead of the traditional therapy procedures. For the demand of new clinic applications, selections of suitable materials for biomedical and clinic applications such as natural polymer, polyvinyl alcohol, polymethacrylate, polyester, polynorbornene, and polymeric resins could be substantially considered and further employed [1-6]. The surface modification could be considered to change the surface characteristic and microenviᄀronment of materials for specific clinic demand [7-8]. High permeability of protecting dressings such as polyvinyl alcohol or polyurethane (PU) or non-woven dressings could be employed for the clinic application of wound management. However, polyurethane or non-woven dressings showed poor tissue anti-adhesion property, which would be a clinic risk for wound managements. Polyvinyl alcohol showed well clinic applications because of good cell or tissue anti-adhesion property.

Chitosan and its oligosaccharides, which are known to possess multiple functional properties, have attracted considerable interest due to their biological activities and potential applications in the biomedical, pharmaceutical, and medical devices [6]. 
Chitosan oligosaccharide (COS) is an oligomer of $\beta$ - $(1 \rightarrow 4)$-linked d-glucosamine. COS can be prepared from the deacetylation and hydrolysis of chitin. COS and its derivatives have been demonstrated to possess several biological activities including anti-inflammation, anti-tumor, tissue regeneration promotion, drug and DNA delivery enhancement, anti-microbial, anti-oxidation and calcium-absorption enhancement [6]. In this study, a series of high permeable chitosan oligosaccharides modified cross-linked polyvinyl alcohol complex foam (COS/cPVACF) dressings with fully open-cell and open-channel microstructures would be designed and prepared. Morphology and characteristics of the resulting chitosan oligosaccharides modified cross-linked polyvinyl alcohol complex foam (COS/cPVACF) dressings could be studied.

\section{Materials and Methods}

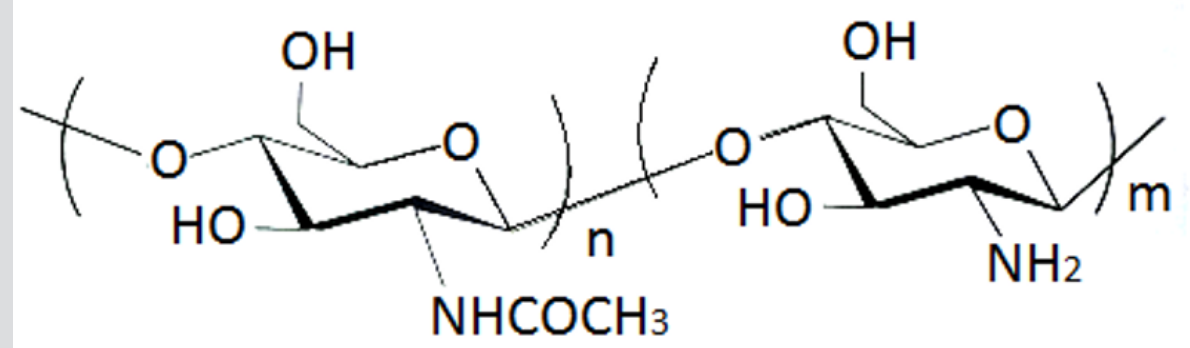

$(n=1-10 m=1-10)$

Scheme 1: Chemical structure of chitosan oligosaccharide for chitosan oligosaccharides modification.

Chitosan oligosaccharide (COS) is a biomaterial obtained by chemical or enzymatic degradation of chitosan derived from shrimp and crab shells [6]. The resulting chitosan oligosaccharide (COS) is composed of 2-10 glucosamines linked by $\beta-1,4$ glycosidic bonds and the molecular weight is up to 3900 Da (Scheme 1). The high medical grade Cenefom PVA raw sheet was employed to prepare a series of new chitosan oligosaccharides modified crosslinked polyvinyl alcohol complex foam (COS/cPVACF) dressings. A combination of novel active molecules cleaning process and a super clean air-foaming process was employed to prepare cross-linked polyvinyl alcohol foam materials with relative low residual formaldehyde contents. Morphology and characteristics of the resulting chitosan oligosaccharides modified cross-linked polyvinyl alcohol complex foam (COS/CPVACF) dressings could be determined by using Fourier transform infrared spectroscopy (FTIR, Spectrum GX, USA) and Scanning electron microscopy (SEM, JSM 6700F, Japan).

\section{Results and Discussions}

The high permeability of protecting dressings could be clinically applied to create an optimal wound healing environment. The cPVA foam could be considered as a good material for the protecting dressings. However, the commercial cPVA foam wound dressings showed relative high formaldehyde residual contents, which would be a risk of wound care. In this work, the designed permeable chitosan oligosaccharide modified cross-linked polyvinyl alcohol complex foam (COS/CPVACF) dressings were prepared by using new materials with low residual formaldehyde contents. A combination of novel active molecules cleaning process and a super clean airfoaming process was designed and employed to prepare crosslinked polyvinyl alcohol foam materials with relative low residual formaldehyde contents $(<1 \mathrm{ppm})$. The selected active molecules such as $\mathrm{O}_{3}$ molecules or supercritical $\mathrm{CO}_{2}$ molecules could be employed [7]. At the same time, permeable microenvironments and architectures could be obtained.

\section{Characteristics of Chitosan Oligosaccharides Modified Cross-Linked Polyvinyl Alcohol Complex Foam (COS/ cPVACF) Dressings}

Characteristics of chitosan oligosaccharides modified crosslinked polyvinyl alcohol complex foam (COS/cPVACF) dressings were determined by FTIR. The FTIR spectrum of cPVA foam can be observed a broad peak at $3300 \mathrm{~cm}^{-1}$ indicating stretching of hydroxyl groups $(-\mathrm{OH})$ and peaks at $2980 \mathrm{~cm}^{-1} 2880 \mathrm{~cm}^{-1}$ are due to $-\mathrm{C}-\mathrm{H}$ stretching vibration. The peak at $1130 \mathrm{~cm}^{1}$ suggested the presence of hydroxymethyl group(-CHOH). The peaks at $103 \mathrm{~cm}^{-1}$ and $918 \mathrm{~cm}^{-1}$ showed C-O stretching vibration of secondary alcohol and $\mathrm{O}-\mathrm{H}$ bend respectively. The peak at $1486 \mathrm{~cm}^{-1}$ was contributed to $-\mathrm{CH} 2$ scissoring and the peak at $1340 \mathrm{~cm}^{-1}$ was contributed to $-\mathrm{OH}$ bending vibration. After modification with chitosan oligosaccharide, the chitosan oligosaccharide modified crosslinked polyvinyl alcohol complex foam (COS/cPVACF) dressings were obtained.

The water permeability of the resulting chitosan oligosaccharide modified cross-linked polyvinyl alcohol complex foam (COS/ cPVACF) dressings were determined by ASTM D4491 standard test methods. The good water permeability was observed in a relative higher level than $80 \%$, which would be contributed by the specific architectures of the COS/CPVACF dressings. Furthermore, the FTIR spectrum of (COS/cPVACF) dressings can be observed to have a series of narrow absorption bands. Amide I band, which is responsible for the splitting of wavenumbers between 1600 to $1500 \mathrm{~cm}^{-1}$, corresponds to the inter-sheet hydrogen bonding due to the $-\mathrm{CHOH}$ group that can be associated to the band peak at 1650 $\mathrm{cm}^{-1}$ and the intra-sheet hydrogen bonding at the $\mathrm{C}=\mathrm{O}$ stretching region with wavenumber $1680 \mathrm{~cm}^{-1}$. This inter- and intra-sheet 
hydrogen bonding could provide a potential application of controlled release system.

\section{Morphology of Chitosan Oligosaccharides Modified Cross-Linked Polyvinyl Alcohol Complex Foam (COS/ cPVACF) Dressings}

The morphology of the resulting cross-linked polyvinyl alcohol foam (CPVAF) dressings and the corresponding chitosan oligosaccharides modified cross-linked polyvinyl alcohol complex foam (COS/CPVACF) dressings were determined by SEM and showed in Figure 1 and Figure 2, respectively. The specific architectures with fully open-cell and open-channel microstructures could be observed. The open-cell microstructures were observed in the range between 80 and100 $\mu \mathrm{m}$ as shown in Figure 2(A). Furthermore, there are several open channels connecting the open cells in the microstructures through the connecting holes. The connecting hole size of open channels were observed in the range between 2 and $5 \mu \mathrm{m}$ as shown in Figure 2(A) and 2(B). The SEM images of chitosan oligosaccharides modified cross-linked polyvinyl alcohol complex foam (COS/CPVACF) dressings showed a remarkably coating on the open-cell microstructures as shown in Figure 3(A) and Figure 3(B). The open-cell microstructures were retained in chitosan oligosaccharides modified cross-linked polyvinyl alcohol complex foam (COS/cPVACF) dressings. The connecting holes and the corresponding open channels were fully covered and filled with chitosan oligosaccharides, which provide a controlled release system of active chitosan oligosaccharide molecules. Furthermore, the Schematic diagram of fully open-cell and open-channel microstructures was provided as shown in Figure 4.

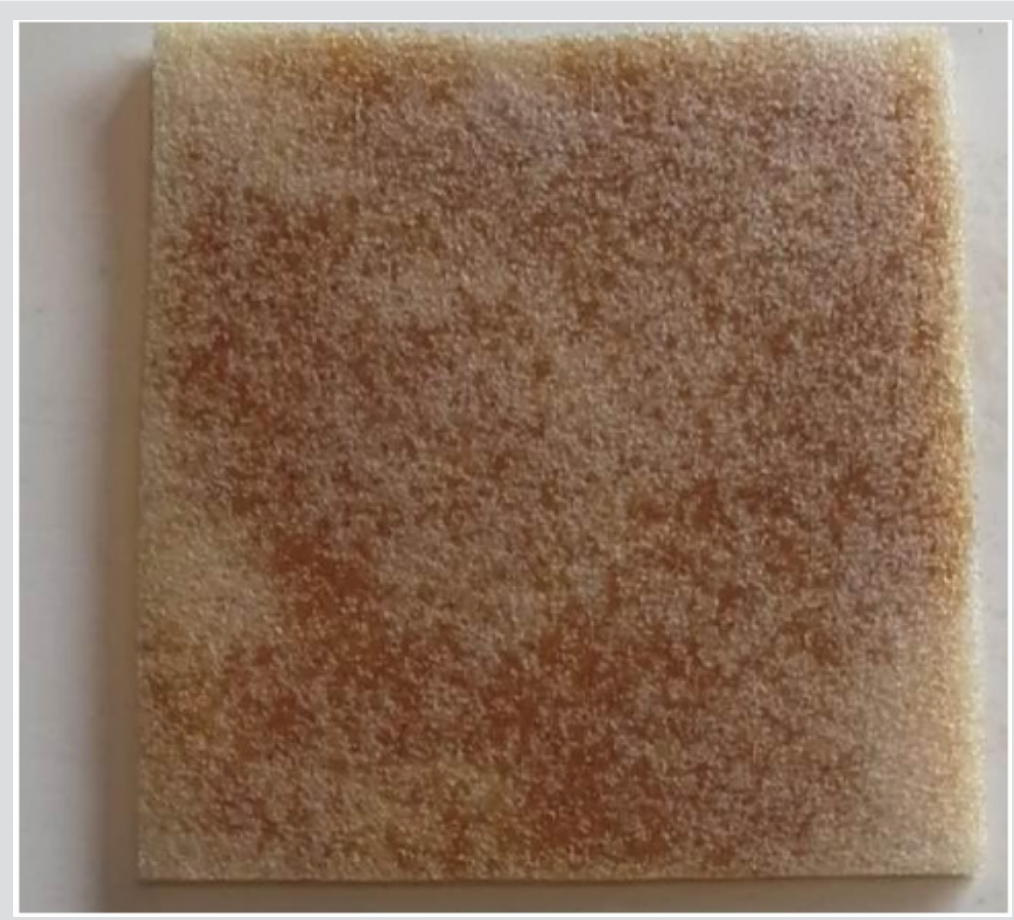

Figure 1: Photo of the designed permeable chitosan oligosaccharide modified cross-linked polyvinyl alcohol complex foam (COS/cPVACF) dressing.

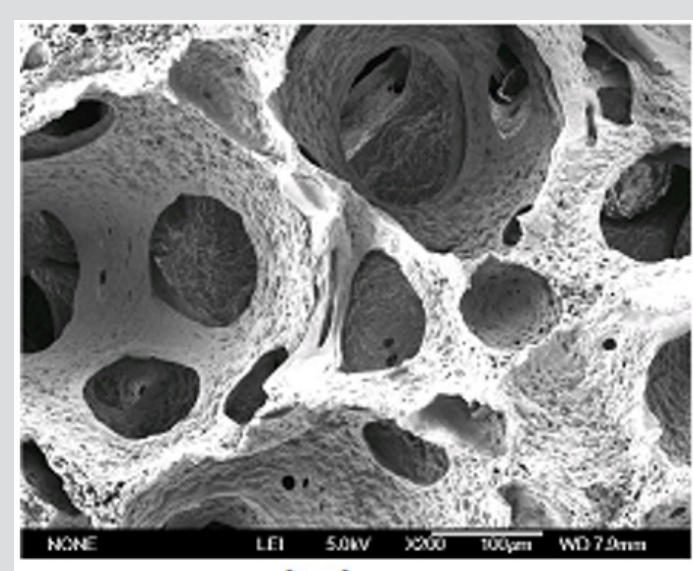

(2A)

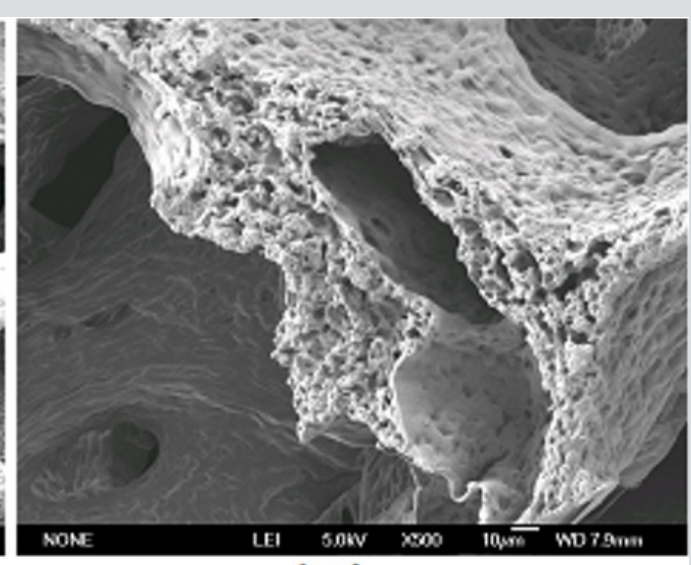

(2B)

Figure 2: Morphology of designed cross-linked polyvinyl alcohol complex foam dressings with fully open-cell and openchannel microstructures, (A) X 200 and (B) $\times 500$. 

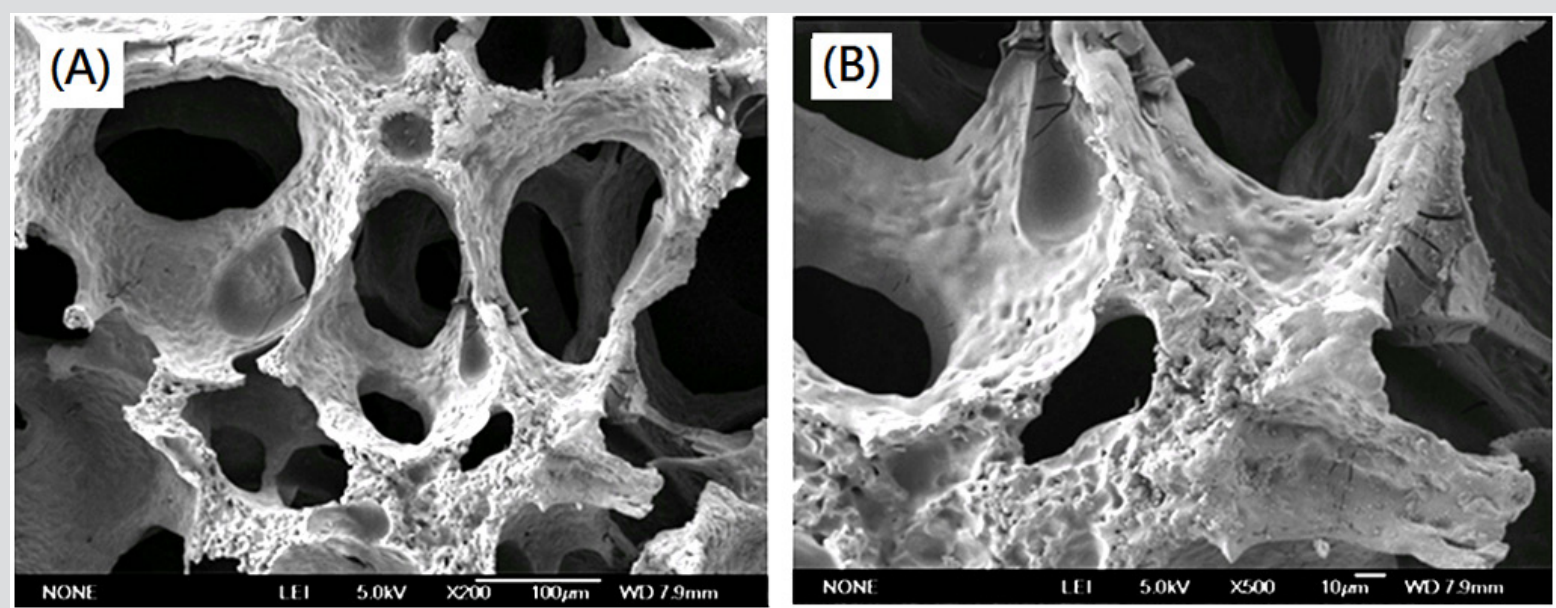

Figure 3: Morphology of designed chitosan oligosaccharide modified cross-linked polyvinyl alcohol complex foam (COS/ cPVACF) dressing with fully open-cell and open-channel microstructures, (A) X 200 and (B) X500.

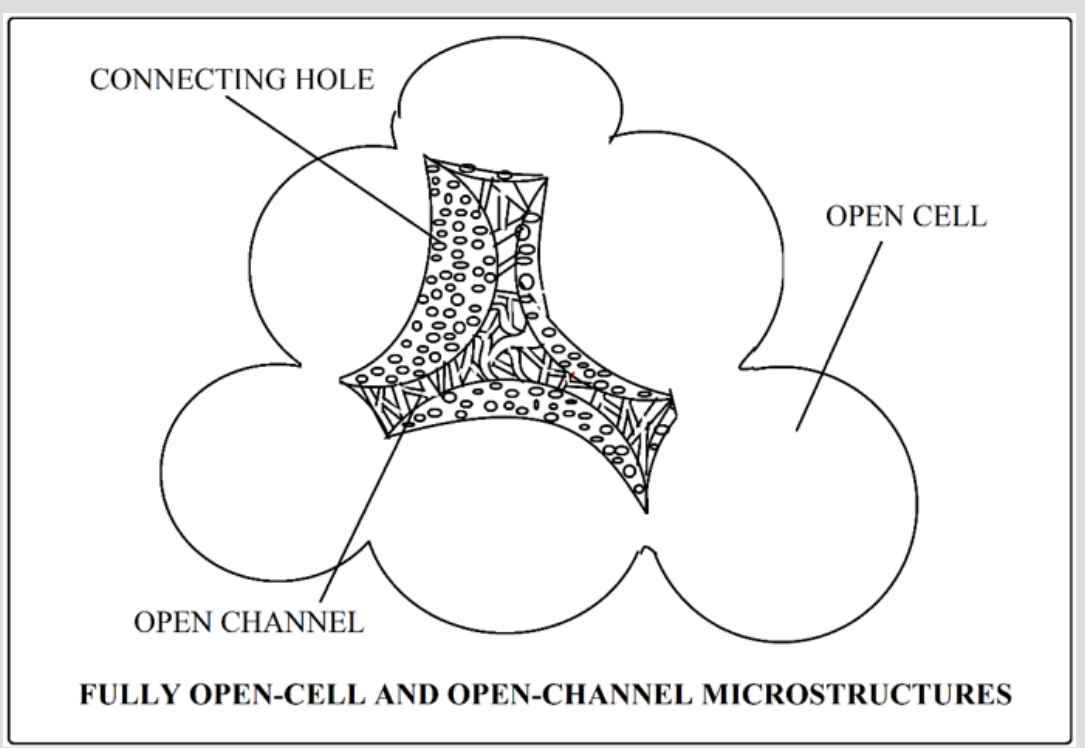

Figure 4: Schematic diagram of fully open-cell and open-channel microstructures.

\section{Conclusion}

The suitable permeable chitosan oligosaccharide modified cross-linked polyvinyl alcohol complex foam (COS/cPVACF) dressing with fully open-cell and open-channel microstructures were successfully prepared and employed to enhance and promote wound healing in wounds, even in acute, chronic and burn wounds.

\section{Acknowledgement}

Authors would like to acknowledge the Taiwan PARSD Pharm Tech. Consulting Ltd Co. for technical supports. The authors also thank for technical assistances of C.-H. Shen.

\section{References}

1. Liaw DJ, Huang CC, Kang ET (2006) Effect of architecture and environments on polymeric molecular assemblies of novel amphiphilic diblock copolynorbornenes with narrow polydispersity via living ringopening metathesis polymerization (ROMP). Journal of Polymer Science Part A: Polymer Chemistry 44(9): 2901-2911.
2. Liaw DJ, Huang CC, Sang HC, Kang ET (1998) Photophysical and solution properties of naphthalene-labeled styrene/N, N-dimethyl maleimido propylammonium propane sulfonate copolymer. Langmuir 14(12): 3195-3201.

3. Zhai G, Toh SC, Tan WL, Kang ET, Neoh KG, et al. (2003) Poly (vinylidene fluoride) with grafted Zwitterionic polymer side chains for electrolyteresponsive microfiltration membranes. Langmuir 19(17): 7030-7037.

4. Liaw DJ, Huang CC, Ju JY (2006) Novel star-like multifunctional polymeric materials with predominant cis microstructures derived from $\alpha$-norbornenyl macromonomer and stable macroinitiator via ring-opening metathesis polymerization and atom transfer radical polymerization. Journal of Polymer Science, Part A: Polymer Chemistry 44: 3382-3392.

5. Huang CC, Yang MJ (2019) Good water absorption and anti-adhesion properties of designed extra thin PVA foam membranes with fully opencell microstructures derived from a super clean air-foaming process with active molecules for minimally invasive surgery. Biomed J Sci \& Tech Res 5(2): 1-3.

6. Chatchai Muanprasat, Varanuj Chatsudthipong (2017) Chitosan oligosaccharide: Biological activities and potential therapeutic applications. Pharmacology \& Therapeutics 170: 80-97. 
7. Li ZF, Kang ET, Neoh KG, Tan KL, Huang CC, et al. (1997) Surface structures and adhesive-free adhesion characteristics of polyaniline films after modification by graft copolymerization. Macromolecules 30(11): 3354-3362.

\section{ISSN: 2574-1241}

DOI: 10.26717/BJSTR.2019.17.003001

Ching Cheng Huang. Biomed J Sci \& Tech Res

(c) (- This work is licensed under Creative

Submission Link: https://biomedres.us/submit-manuscript.php
8. Zhai G, Toh SC, Tan WL, Kang ET, Neoh KG, et al. (2003) Poly (vinylidene fluoride) with grafted Zwitterionic polymer side chains for electrolyteresponsive microfiltration membranes. Langmuir 19(17): 7030-7037.

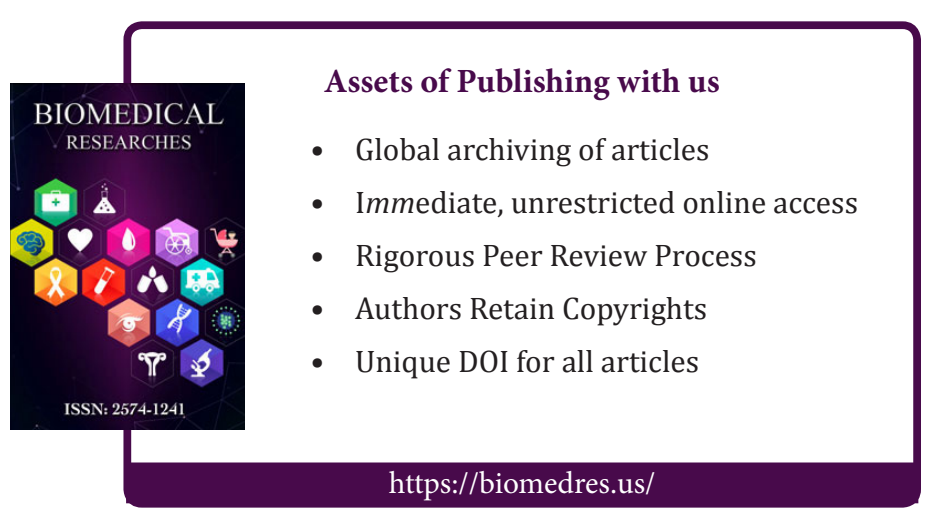

